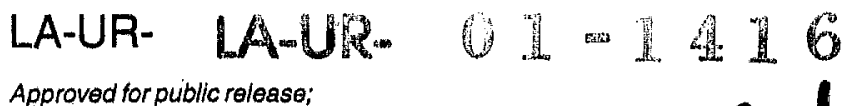 distribution is unlimited.
}

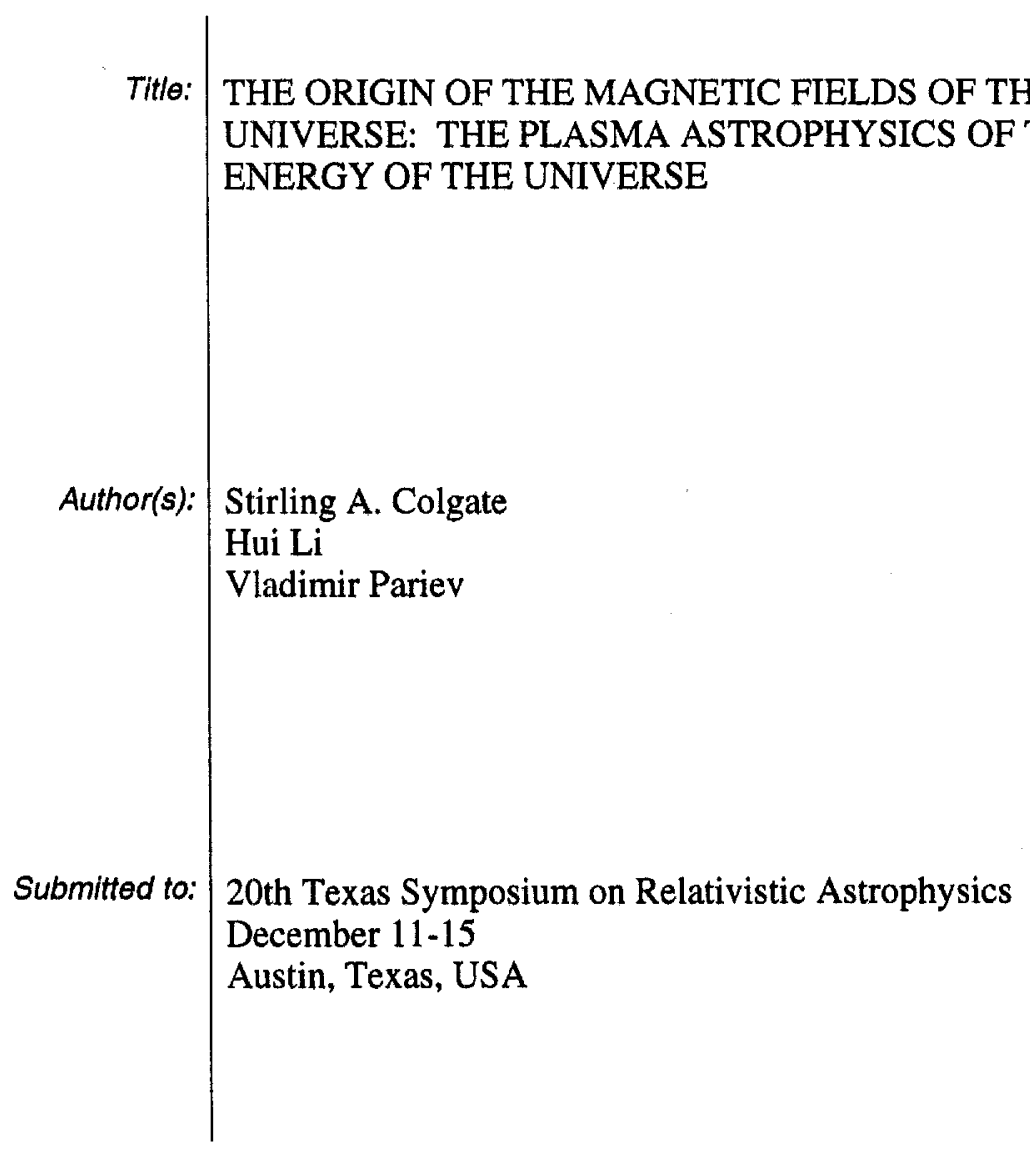

\section{Los Alamos \\ NATIONAL LABORATORY}

Los Alamos National Laboratory, an affirmative action/equal opportunity employer, is operated by the University of Californla for the U.S. Department of Energy under contract W-7405-ENG-36. By acceptance of this article, the publisher recognizes that the U.S. Government retains a nonexclusive, royalty-Iree license to publish or reproduce the published form of this contribution, or to allow others to do so, for U.S. Government purposes. Los Alamos National Laboratory requests that the pub $\equiv$ identify this article as work pertormed under the auspices of the U.S. Department of Energy. Los Alamos National Laboratory s: $=$ y supports academic freedom and a researcher's right to publish; as an institution, however, the Laboratory does not endorse the viewpoint of a publication or guarantee its technical correctness. 


\title{
The Origin of the Magnetic Fields of the Universe: The Plasma Astrophysics of the Free Energy of the Universe
}

\author{
Stirling A. Colgate*, Hui Li* and Vladimir I. Pariev*ł \\ * Theoretical Astrophysics Group, T6, Los Alamos National Laboratory, \\ Los Alamos, New Mexico 87545, USA \\ ${ }^{\dagger}$ Steward Observatory, 933 N. Cherry Ave., Tucson, AZ 85721, USA \\ $\ddagger$ P.N. Lebedev Physical Institute, Leninsky Prospect 53, Moscow 117924, Russia
}

\begin{abstract}
The largest accessible free energy in the universe is almost certainly the binding energy of the massive central black hole $(\mathrm{BH})$ of nearly every galaxy. We have calculated one mechanism that produces this characteristic mass, $10^{8} M_{\odot}$, by initiating a Rossby vortex dominated accretion disk at a critical thickness, $\sim 100 \mathrm{~g} \mathrm{~cm}^{-2}$, in the development of the flat rotation curve of nearly every galaxy. We have simulated how an $\alpha-\Omega$ dynamo should work due to star-disk collisions and plume rotation. The back reaction of this saturated dynamo may convert almost all the accretion energy into a single force-free magnetic field helix. This helix and field energy is then distributed as a quasi-static, hydrodynamically stable, Poynting flux configuration, filling the intergalactic space with a magnetized plasma. This energy and flux also explains the Faraday rotation maps of AGN in clusters. This energy density is $\sim 10^{3}$ times the virial energy of a galactic mass of baryonic matter in the combined gravity of dark and baryonic matter on the galaxy scale and before and during galaxy formation. This extra galactic energy density should affect subsequent galaxy formation. This possibly explains why the large extra galactic mass of gas in both clusters and the walls has not subsequently formed further galaxies. Also the reconnection of this magnetic field during a Hubble time provides enough energy to maintain the extra galactic cosmic ray spectrum.
\end{abstract}

The total energy released by the growth of supermassive black holes at the center of nearly every galaxy is large and can be comparable or even larger than that emitted by stars in the universe. Recent observations suggest that radiation from Active Galactic Nuclei (AGNs or quasars) might account for only 10\% of this energy [1]. Where did the rest of the energy go? We propose that a major fraction of this energy has been converted into magnetic energy and stored in the large scale magnetic fields primarily external to each galaxy, in galaxy clusters and "walls". We have envisioned a sequence of key physical processes that describes this energy 
flow [2-4].

The interpretation of Faraday rotation measure maps of jets and radio lobes of radio galaxies within galaxy clusters has revealed ordered or coherent regions, $L_{\text {mag }} \sim 50-100 \mathrm{kpc}\left(\sim 3 \times 10^{23} \mathrm{~cm}\right)$, that are populated with large, $\sim 30 \mu \mathrm{G}$ magnetic fields [5-7]. The magnetic energy of these coherent regions is $L_{\text {mag }}^{3}\left(B^{2} / 8 \pi\right) \sim 10^{59}-10^{60} \mathrm{ergs}$, and the total magnetic energy over the whole cluster ( $\sim 1 \mathrm{Mpc}$ across) is expected to be even larger. Understanding the origin and role of these magnetic fields is a major challenge to plasma astrophysics.

A sequence of physical processes that are responsible for the production, redistribution and dissipation of these magnetic fields is proposed. These fields are associated with single Active Galactic Nucleus (AGN) within the cluster and therefore with all galaxies during their AGN (Active Galactic Nucleus or Quasar) phase, simply because only the central supermassive black holes $\left(\sim 10^{8} M_{\odot}\right)$ formed during the AGN phase have an accessible energy of formation, $\sim 10^{61} \mathrm{ergs}$, that can account for the magnetic field energy budget. An $\alpha-\Omega$ dynamo process has been

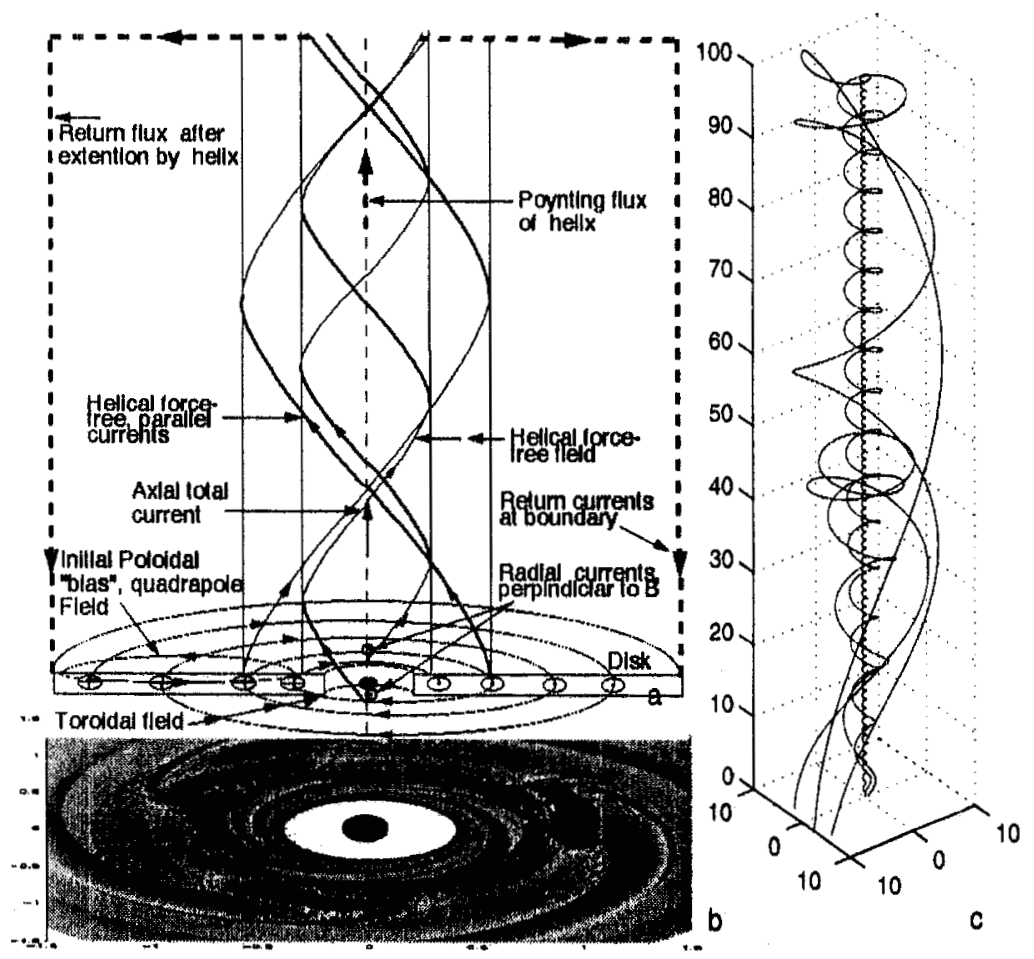

FIGURE 1. The helix generated by the dynamo in the disk. The foot points of the external quadrupole field lines, (a), generated by the dynamo, are attached to the surface of the disk (b). In the conducting low density plasma, $\beta \ll 1$, these field lines are wrapped into a force-free helix. This helix extends from the disk carrying flux and energy, a Poynting flux. (c). 
proposed that operates in an accretion disk around a black hole. Such an accretion disk is formed in the central part of the collapsing cloud of barionic matter, which forms the galaxy. When the surface density of barionic ellipsoid exceeds the value $\sim 100 \mathrm{~g} \mathrm{~cm}^{-2}$ large enough for a disk to contain its heat against radiative cooling, the mechanism of outward angular momentum transport by Rossby vortices sets in and allows the barionic gas to reach the center and to form $a \sim 10^{8}$ solar masses black hole. We have found a global nonaxisymmetric instability to exist in thin disks that can excite large scale vortices in the disk. Such vortices are shown to transport angular momentum outward efficiently [8].

The disk rotation naturally provides a large winding number, $\sim 10^{11}$ turns, sufficient to make both large gain and large flux. The helicity of the dynamo can be generated by the differential plume rotation derived from star-disk collisions. This helicity generation process has been demonstrated in the laboratory and the dynamo gain was simulated numerically. A liquid sodium analog of the dynamo is being built. Unlike dynamos driven by hydrodynamic or hydromagnetic instabilities in the accretion disk, star-disk collision driven dynamo has no problems with $\alpha$ quenching and can result in the magnetic fields stronger than equipartition value with the thermal pressure in the disk. Speculations are that the back reaction of the saturated dynamo will lead to the formation of a force-free magnetic helix, which will carry the energy and flux of the dynamo away from the accretion disk and redistribute the field within the clusters and walls. Such a helix is shown schematically in Figure 1a and calculated with the force-free field, Grad-Shafranov equations with a cylindrical boundary, Figure 1c. The magnetic reconnection of a small fraction of this energy logically is the source of the AGN luminosity, and the remainder of the field energy should then dominate the free energy of the presentday universe. The reconnection of this intergalactic field during a Hubble time is the only sufficient source of energy necessary to produce an extragalactic cosmic ray energy spectrum as observed in our Galaxy, and at the same time allow this spectrum to escape to the voids faster than the time of the Gruneisen-ZatsepinKuzmin loss due to black body radiation photons.

\section{REFERENCES}

1. Richstone, D.O., Ajhar, E.A., Bender, R. et al., Nature 395, 14 (1998).

2. Colgate, S.A., and Li, H., Astrophys. Space Sci. 264, 357 (1999)

3. Colgate, S.A., and Li, H., IAUS 195, ASP Conf. Series 334, eds. P.C.H. Martens and S. Tsurta, 1999

4. Colgate, S.A., Li, H., Pariev, V.I., Physics of Plasmas, in press (2001)

5. Taylor, G.B., and Perley, R.A., Astrophys. J. 416, 554 (1993).

6. Clarke, T.E., Kronberg, P.P., and Böhringer, H. Astrophys. J. Letters 547, L111 (2001).

7. Kronberg, P.P., Prog. Phys. 57, 325 (1994).

8. Li, H., Colgate, S.A., Wendroff, B., and Liska, R., Astrophys. J., in press (2001). 